\title{
Image Processing of Energy Filtered Transmission Electron Microcopy Maps in Order to Extract Nano Scale Magnetic Properties of CoCr-based Magnetic Thin Films
}

\author{
Jafar F. Al-Sharab ${ }^{1}$, and Mohammed Benalla ${ }^{1}$ \\ 1. Department of Engineering Technology, Northwestern State University, Natchitoches, LA, USA
}

The design of low noise media with enhanced thermal stability is strongly dependent upon understanding the chemistries of magnetic thin films at the nano-scale and the knowledge of basic magnetic properties such as mangetocrystalline anisotropy, ku and saturation magnetization, Ms. The magnetic anisotropy plays a dominant role in determining magnetic energy of recording media. Measurement of this property at the nanoscale is extremely difficult. In this research, we utilize the spectroscopy imaging and image processing techniques in order to extract magnetic properties from Energy Filtered Transmission Electron Microscopy (EFTEM) elemental maps. This techniques is demonstrated for CoCr-based magnetic thin films with $\mathrm{Cr}$ underlayer

Samples of $\mathrm{Co}_{80} \mathrm{Cr}_{16}(\mathrm{Pt}$ and $\mathrm{Ta})$ thin film media were produced by d.c. magnetron sputtering onto $20 \mathrm{~nm}$ thick CrMo (15 at. \% Cr) seed layer with an amorphous-NiP-plated aluminum substrate. Plan view as well as cross section samples for EFTEM were prepared by back-thinning with the final ion milling produced by a single gun at $4 \mathrm{keV}$ and $12^{\circ}$. The EFTEM was performed using a $300 \mathrm{kV}$ Philips CM30, with $\mathrm{LaB}_{6}$ cathode and a Gatan Image Filter (GIF). Additional experimental details are presented elsewhere [1,2]. Specialized DigitalMicrograph custom scripts were used to process collected images and to extract accurate pixel intensities due to $\mathrm{Co}$ and $\mathrm{Cr} \mathrm{L}_{2,3}$ core-loss excitations. Additionally, Fourier Transform analysis where used defect analysis in high resolution TEM imaging.

EFTEM data with a $1 \mathrm{~nm}$ spatial resolution were utilized to measure the average $\mathrm{Cr}$ content inside the grains. In collecting EFTEM images, the beam was defocused in order to obtain at least 300 grains for statically analysis. Additionally, each pixel has 10,000 to 12,000 counts in order to have strong signal to noise ratio and to reach sufficient readings for statistical analysis. Quantification of the $\mathrm{Cr}$ grain boundary profiles utilized intensity ratio images where the $\mathrm{Cr}$ map was divided by the Co map to minimize the effect of diffraction contrast, sample thickness variation, and illumination differences. The quantitative analyses were simplified by experimental observations from energy dispersive spectroscopy, that the Ta and Pt atoms have homogeneous distribution. Nano probe technique.

The $\mathrm{K}_{\mathrm{u}}$ was estimated as a function of $\mathrm{Cr}$ concentration from single crystal CoCr-based media with similar composition and film thickness. This method allows the estimation of the magnetic energy, $\mathrm{K}_{\mathrm{u}} \mathrm{V}$ ( $\mathrm{V}=$ volume of grain) distribution of CoCr-based media, which determines the percentage of thermally stable grains or domains. The microstructure characterization and elemental mapping were conducted using transmission electron microscopy (TEM) and energy-filtered electron transmission microscopy (EFTEM), Figure 1.

Magnetocrystalline anisotropy and magnetic energy of poly crystalline CoCrTa magnetic thin film media were estimated from EFTEM compositional data, Figure 1. Distributions of $\mathrm{K}_{\mathrm{u}}$ and $\mathrm{K}_{\mathrm{u}} \mathrm{V}$ were reported. Data analysis shows that the number of thermally stable grains has increased over $40 \%$ with 
increasing the substrate temperature up to 250C [2]. The obtained results will be of great benefit for micro/nano magnetic modeling for both longitudinal and perpendicular magnetic media. [3]

\section{References:}

[1] Jafar F. et al, Eur. Journal of applied physics, 42, 125-128 (2008)

[2] J.F. Al-Sharab, "The effect of alloying and process conditions of the microstructure and magnetic properties of $\mathrm{CoCr}(\mathrm{PtTa})$ longitudinal recording media", Ph.D. Dissertation in, Material Science and engineering, Vanderbilt University, Nashville, TN,USA.(2003)

[3] The EFTEM data were collected at Oak Ridge National Laboratory with Dr. James Bentley

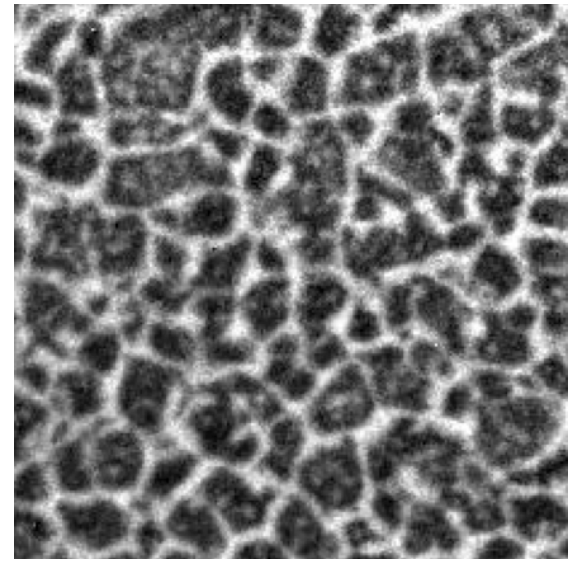

(a)

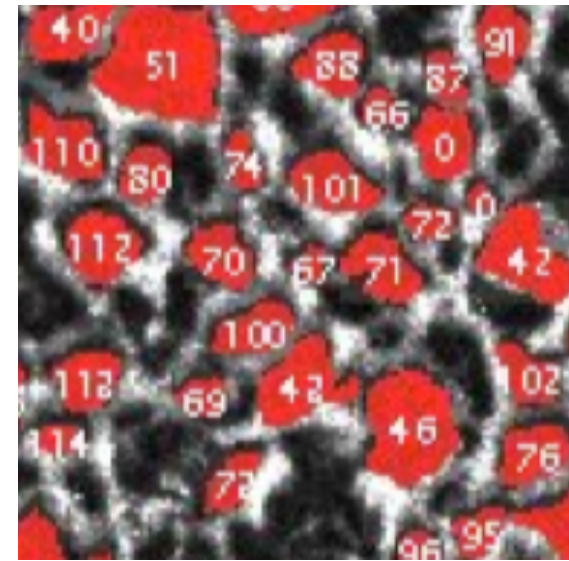

(b)

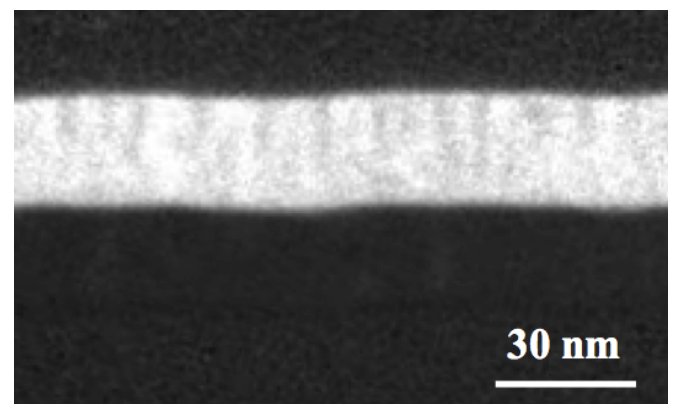

(c)

Figure 1. ETEM images of recording media, (a) EFTEM image showing high Cr concentration at grain boundaries, (b) EFTEM image $(100 \times 100 \mathrm{~nm})$ with masked grains for accurate chemical analysis, and (c) cross-sectional ETEM image showing the thickness of the $\mathrm{CoCr}$ magnetic thin layer (appears in white) 